\title{
C- 3 SUBSTITUTED LAWSONEMONOXIMATES OF HOLMIUM(III): SYNTHESIS, CHARACTERIZATION, AND ANTIMICROBIAL ACTIVITY
}

\author{
S. B. Jagtap ${ }^{1}$, S. G. Joshi², G. M. Litake', V. S. Ghole*1, and B. A. Kulkarni1 \\ 1 Department of Chemistry, University of Pune, Pune - 411 007. India. \\ ${ }^{1}$ Department of Pathology \& Microbiology, D. S. H. Medical College, Pune-411004. \\ Fax: + 91205651728 <ysghole@chem.unipune.ernet.in>
}

\begin{abstract}
A series of five new metal complexes of Ho(III) with C-3 substituted derivatives of lawsonemonoxime (2-hydroxy-1,4-naphthalenedione-1-oxime) were synthesized. The compounds were characterized by melting point, elemental analysis, IR spectroscopy and magnetic susceptibility. The antimicrobial activity of the compounds were determined by disk diffusion method and broth micro-dilution techniques using Mueller Hinton medium against the following organisms: $S$. aureus ATCC 6538P, Klebsiella pneumoniae, NCTC 418, Pseudomonas aeruginosa ATCC 27833, Salmonella typhimurium ATCC $23564, E$. coli U 1777, E. coli HB101, Proteus morganii NCIM 2860, Providencia stuartii NCIM 2799 and Acinetobacter baumannii U 24. The chelates of Ho(III) with lawsonemonoxime and Ho(III) with 3bromolawsonemonoxime showed a variable antimicrobial activity against all organisms tested except Pseudomonas and Klebsiella spp. S. aureus was found more sensitive to all ligands and chelates tested; but the MIC values of chelates were considerably less; thus having more antimicrobial effect.
\end{abstract}

\section{INTRODUCTION}

Antimicrobial or antineoplastic activity of lawsone was studied by Lime et al [1].

Tripathi et al [2] showed that lawsone exhibits fungicidal activity. Anderson et al [3] studied phthiocol which is the quinone compound associated with certain bacteria. Some oxime derivatives of lawsone and their chelates showed antimicrobial activity. Mhaske et al [4] have reported the antimicrobial activities of 3-nitrolawsonemonoxime, 3-aminolawsonemonoxime and their bivalent metal chelates where as Dandawate [5] and Gaikwad [6] studied the chelates of some rare earths with lawsonemonoxime derivatives. Joshi et al [7] have reported on rare earth studies where as Shen et al [8] studied lanthanide metal complexes for antimicrobial activities. But the chelates of Ho(III) with lawsonemonoximes (figure 1) and their antimicrobial activities are being reported for the first time in the present studies.

A

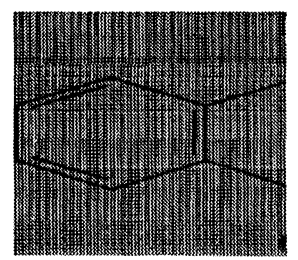

B

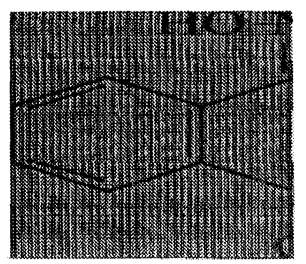

Figure 1: (A) Structure of lawsonemonoxime derivatives

\begin{tabular}{ll}
$\mathrm{R}$ & Ligand \\
\hline $\mathrm{H}$ & Lawsonemonoxime (HL1) \\
$\mathrm{CH}_{3}$ & Phthiocolmonoxime (HL2) \\
$\mathrm{Cl}$ & 3-Chlorolawsonemonoxime (HL3) \\
$\mathrm{Br}$ & 3-Bromolawsonemonoxime (HL4) \\
$\mathrm{I}$ & 3-lodolawsonemonoxime (HL5)
\end{tabular}

(B): General structure of the ligating system

\section{MATERIALS AND METHODS \\ Preparation of the ligands}

All the chemicals and solvents used were of analytical grade. Lawsone (2-hydroxy-1,4naphthalenedione), dichlone (2,3-dichloro-1,4-naphthalenedione) and menadione (2-methyl-1,4naphthalenedione) were purchased from Fluka (Germany). Phthiocol was synthesized from menadione by Fieser's method [9]. The 3-chlorolawsone was prepared from dichlone. All the ligands (figure 1) used (lawsonemonoxime derivatives) were synthesized by treating the solution of a lawsone derivative with solution of hydroxylamine hydrochloride. The entire mixture was heated at $60^{\circ} \mathrm{C}$, cooled and then neutralized with hydrochloric acid (2M) causing precipitation of the corresponding lawsone-1-oxime derivative. The melting points were recorded after recrystallization of the ligands from methanol. The compounds were tested for solubility in water, methanol, DMF, DMSO and acetonitrile.

\section{Preparation of the Chelates}

To a hot solution of $3.0 \mathrm{mmol}$ of ligand (table 1), $(0.568 \mathrm{~g}$ of lawsonemonoxime, $0.60 \mathrm{~g}$ of phthiocolmonoxime, $0.671 \mathrm{~g}$ of 3-chlorolawsonemonoxime, $0.804 \mathrm{~g}$ of 3-bromolawsonemonoxime and $0.945 \mathrm{~g}$ of 3-iodolawsonemonoxime) in $25 \mathrm{~mL}$ of ethanol, an aqueous solution of $1.00 \mathrm{mmol}$ of 
metal(III)chloride hexahydrate $\left(0.379 \mathrm{~g}\right.$ of $\left.\mathrm{HoCl}_{3} .6 \mathrm{H}_{2} \mathrm{O}\right)$ was added. The $\mathrm{pH}$ of the mixture was adjusted to 5.5 to 6.5 using aqueous ammonia $(1: 20 \mathrm{v} / \mathrm{v})$. The mixture was refluxed for $3 \mathrm{~h}$ and then cooled overnight. The precipitate was filtered off, washed with water followed by hot methanol and dried in vacuum over fused $\mathrm{CaCl} 2$ at ambient temperature. A solubility of the complexes was tested in methanol, water, DMF, DMSO and acetonitrile.

The carbon, hydrogen analysis and residue $\left(\mathrm{Ho}_{2} \mathrm{O}_{3}\right)$ were determined using a Hosli- Holland $\mathrm{C}, \mathrm{H}$ Analyzer. The magnetic studies were carried out at room temperature by the Faraday technique using mercury(II) tetrathiocyanatocobaltate as calibrant. The infrared spectra were recorded in nujol mulls on a Perkin- Elmer FTIR Spectrophotometer (Model 1600, range 4000- $450 \mathrm{~cm}^{-1}$ ).

Table 1. Elemental analysis and some physical properties of the ligands having antimicrobial activity.

\begin{tabular}{|l|l|c|c|c|c|c|}
\hline Ligand & \multicolumn{2}{|c|}{ Colour } & M.p. & Yield & Elemental & \multicolumn{2}{c|}{$\begin{array}{c}\text { Analysis } \\
\text { (calc.) }\end{array}$} \\
\hline & & $\left.{ }^{\circ} \mathrm{C}\right)$ & $(\%)$ & $\%$ of C & $\%$ of H & $\%$ of N \\
\hline HL1 & Yellow & 180 & 85 & $\begin{array}{c}63.17 \\
(65.49)\end{array}$ & $\begin{array}{c}3.72 \\
(3.70)\end{array}$ & $\begin{array}{c}7.45 \\
(7.41)\end{array}$ \\
\hline HL2 & Pale yellow & 200 & 95 & $\begin{array}{c}65.35 \\
(65.02)\end{array}$ & $\begin{array}{c}4.41 \\
(4.43)\end{array}$ & $\begin{array}{c}6.93 \\
(6.91)\end{array}$ \\
\hline HL3 & Glossy greenish yellow & 188 & 94 & $\begin{array}{c}53.46 \\
(53.69)\end{array}$ & $\begin{array}{c}2.66 \\
(2.68)\end{array}$ & $\begin{array}{c}6.23 \\
(6.26)\end{array}$ \\
\hline HL4 & Pale greenish yellow & 192 & 90 & $\begin{array}{c}43.49 \\
(44.77)\end{array}$ & $\begin{array}{c}2.20 \\
(2.24)\end{array}$ & $\begin{array}{c}5.05 \\
(5.22)\end{array}$ \\
\hline HL5 & Pale greenish yellow & 158 & 88 & $\begin{array}{c}37.98 \\
(38.10)\end{array}$ & $\begin{array}{c}2.10 \\
(1.90)\end{array}$ & $\begin{array}{c}4.42 \\
(4.44)\end{array}$ \\
\hline
\end{tabular}

M.p., melting point, $\mathrm{HL} 1,\left(\mathrm{C}_{10} \mathrm{H}_{7} \mathrm{O}_{3} \mathrm{~N}\right)$; $\mathrm{HL} 2,\left(\mathrm{C}_{11} \mathrm{H}_{9} \mathrm{O}_{3} \mathrm{~N}\right)$; $\mathrm{HL} 3,\left(\mathrm{C}_{10} \mathrm{H}_{6} \mathrm{O}_{3} \mathrm{~N} . \mathrm{Cl}\right) ; \mathrm{HL} 4,\left(\mathrm{C}_{10} \mathrm{H}_{6} \mathrm{O}_{3} \mathrm{~N} . \mathrm{Br}\right)$; HL5, $\left(\mathrm{C}_{10} \mathrm{H}_{6} \mathrm{O}_{3}\right.$ N.I $)$.

\section{Antimicrobial activity of the compounds}

The antimicrobial activity was determined by the disk diffusion method[10] against the following organisms: Staphylococcus aureus ATCC 6538P, Klebsiella pneumoniae NCTC 418, Pseudomonas aeruginosa ATCC 27833, Salmonella typhimurium ATCC 23564, E. coli U1777, E. coli HB101, Proteus morganii NCIM 2860, Providencia sturtii NCIM 2799, and Acinetobacter baumannii U24.

In brief, Mueller Hinton agar (HiMedia Lab, India) was prepared as per instructions of the manufacturer and plates were poured around $56^{\circ} \mathrm{C}$ to set and then placed in the incubator at $37^{\circ} \mathrm{C}$ for overnight to test the sterility of medium. Meanwhile, an overnight nutrient broth culture of each strain was taken and diluted to reach O.D. of 0.5 MacFarland standard with nutrient broth, of which $50 \mu \mathrm{L}$ was inoculated on plate and spreaded with glass spreader. After $1 \mathrm{~h}$ the disks (prepared from Whatman No 42, $6 \mathrm{~mm}$ diameter) impregnated with the compounds (prepared in DMSO to water 3:1) were applied and the plates incubated at $37^{\circ} \mathrm{C}$ for $48 \mathrm{~h}$. The zones of inhibition of growth around disks were measured at the intervals of 18,24 and $48 \mathrm{~h}$. The method was applied to all strains selected and repeated twice.

\section{Determination of MICs}

The minimum inhibitory concentrations (MICs) were determined using broth microdilution technique. Serial two fold dilutions of compounds ranging the concentrations from $1024 \mu \mathrm{g}$ through $32 \mu \mathrm{g} /$ $\mathrm{mL}$ were prepared in nutrient broth. The inoculum of $50 \mu \mathrm{L}$ of nutrient broth culture, having O.D. of 0.5 MacFarland standard was inoculated into sterile bumper test tubes $(2.5 \mathrm{~cm} \times 15 \mathrm{~cm})$ containing $5 \mathrm{~mL}$ of Mueller Hinton broth containing above concentrations of compounds. The tubes were incubated at $37^{\circ} \mathrm{C}$ for $48 \mathrm{~h}$ and observed for any visible growth at the intervals of 18,24 and $48 \mathrm{~h}$. The MIC was defined as the lowest concentration of compound in the medium, showing complete inhibition of visible growth.

\section{RESULTS AND DISCUSSION}

The interaction of the ligands (table 1) with metal salts in 1:3 molar ratio in ethanol yielded stable solid complexes corresponding to the molecular composition supported by elemental analysis is $\operatorname{HoL}_{3}\left(\mathrm{H}_{2} \mathrm{O}\right)_{2}$ (where $\mathrm{L}=$ anion of the corresponding ligands). All the complexes (table 2) are soluble in donor solvents such as DMSO and DMF, moderately soluble in solvents like methanol and acetonitrile and insoluble in inert solvents like $n$-hexane, benzene,1,4-dioxane etc. All the ligands were soluble in methanol, water, DMF, DMSO and acetonitrile. 
Table 2. Elemental analysis and physical properties of metal chelates exhibiting some antimicrobial activity.

\begin{tabular}{|l|l|l|l|l|l|l|l|l|}
\hline Compd & Colour & $\begin{array}{l}\text { Decom. } \\
\text { Temp. }\end{array}$ & Yield & \multicolumn{3}{|l|}{$\begin{array}{l}\text { Elemental } \\
\text { (Calc.) }\end{array}$} & $\begin{array}{l}\text { Analysis } \\
\text { found }\end{array}$ \\
\hline & & $(\mathrm{OC})$ & $(\%)$ & $\% \mathrm{C}$ & $\% \mathrm{H}$ & $\% \mathrm{~N}$ & $\% \mathrm{M}$ & \\
\hline Ho1 & Yellow & 290 & 66.68 & $\begin{array}{l}46.79 \\
(47.07)\end{array}$ & $\begin{array}{l}3.00 \\
(2.90)\end{array}$ & $\begin{array}{l}5.36 \\
(5.49)\end{array}$ & $\begin{array}{l}21.38 \\
(21.55)\end{array}$ & 10.08 \\
\hline Ho2 & Orange & 236 & 67.47 & $\begin{array}{l}48.77 \\
(49.08)\end{array}$ & $\begin{array}{l}3.12 \\
(3.49)\end{array}$ & $\begin{array}{l}5.13 \\
(5.30)\end{array}$ & $\begin{array}{l}20.36 \\
(20.42)\end{array}$ & 10.30 \\
\hline Ho3 & Turmeric yellow & 296 & 68.54 & $\begin{array}{l}41.01 \\
(41.48)\end{array}$ & $\begin{array}{l}2.52 \\
(2.20)\end{array}$ & $\begin{array}{l}4.81 \\
(4.84)\end{array}$ & $\begin{array}{l}18.79 \\
(18.98)\end{array}$ & 10.23 \\
\hline Ho4 & Turmeric yellow & 290 & 69.37 & $\begin{array}{l}36.02 \\
(35.96)\end{array}$ & $\begin{array}{l}2.25 \\
(1.99)\end{array}$ & $\begin{array}{l}3.98 \\
(4.19)\end{array}$ & $\begin{array}{l}16.23 \\
(16.46)\end{array}$ & 10.27 \\
\hline Ho5 & Turmeric yellow & 268 & 67.45 & $\begin{array}{l}31.79 \\
(31.52)\end{array}$ & $\begin{array}{l}2.01 \\
(1.68)\end{array}$ & $\begin{array}{l}3.90 \\
(3.68)\end{array}$ & $\begin{array}{l}14.20 \\
(14.43)\end{array}$ & 10.12 \\
\hline
\end{tabular}

$\mu$ eff. is magnetic moment (BM). Hol, $\mathrm{Ho}(\mathrm{III}) \cdot\left(\mathrm{C}_{1} \mathrm{OH}_{6} \mathrm{O}_{3} \mathrm{~N}\right)_{3} .2 \mathrm{H}_{2} \mathrm{O} ; \mathrm{Ho} 2$, $\mathrm{Ho}$ (III) $\left(\mathrm{C}_{11} \mathrm{H}_{8} \mathrm{O}_{3} \mathrm{~N}\right)_{3} .2 \mathrm{H}_{2} \mathrm{O}$; $\mathrm{Ho} 3$, $\mathrm{Ho}$ (III) $\left(\mathrm{C}_{10} \mathrm{H}_{5} \mathrm{O}_{3} \mathrm{NCl}\right)_{3} .2 \mathrm{H}_{2} \mathrm{O}$; Ho4, $\mathrm{Ho}$ (III) $\left(\mathrm{C}_{10} \mathrm{H}_{5} \mathrm{O}_{3} \mathrm{~N} . \mathrm{Br}\right)_{3} .2 \mathrm{H}_{2} \mathrm{O} ; \mathrm{Ho}$, Ho(III) $\left(\mathrm{C}_{10} \mathrm{H}_{5} \mathrm{O}_{3} \mathrm{~N}_{\text {II }}\right)_{3} .2 \mathrm{H}_{2} \mathrm{O}$.

Selected IR bands of the ligands and complexes are shown in table 3. Ligands exhibit a medium broad band at $3100-3600 \mathrm{~cm}^{-1}$ which is due to the $(\mathrm{O}-\mathrm{H})$ vibration of oximino and phenolic hydroxyls. On complexation, this band is further broadened due to overlap of the $(\mathrm{O}-$ $\mathrm{H})$ stretching frequency of coordinated molecules[5]. The $(\mathrm{C}=\mathrm{O})$ stretching frequency at $1600-1630 \mathrm{~cm}^{-1}$ found in the IR spectra of ligands is shifted to a lower frequency region by 20 $30 \mathrm{~cm}^{-1}$ after complexation. This indicates redistribution of electron density in the quinonoid ring i.e. weakening of bond.

The band at $1570-1590 \mathrm{~cm}^{-1}$ in the spectra of ligands is assigned to $(\mathrm{C}=\mathrm{N})$ vibrations, it is shifted to lower frequency by $40-70 \mathrm{~cm}^{-1}$ in the complexes (table 3 ), which indicates a coordination through the oximino nitrogen. A quinone absorption is observed at 1285-1295 $\mathrm{cm}^{-1}$.

Table 3. Selected IR bands of ligands and metal chelates showing antimicrobial activity $\left(\mathrm{cm}^{-1}\right)$.

\begin{tabular}{|l|l|l|l|l|l|l|l|l|}
\hline Compd & $(\mathrm{O}-\mathrm{H})$ & $(\mathrm{C}=\mathrm{O})$ & $(\mathrm{C}=\mathrm{N})$ & $\begin{array}{l}\text { Quinone } \\
\text { absorption }\end{array}$ & $(\mathrm{C}-\mathrm{O})$ & $(\mathrm{N}-\mathrm{O})$ & $(\mathrm{C}-\mathrm{X})$ & $(\mathrm{Ho}-\mathrm{O})$ \\
\hline HL1 & 3362,3155 & 1630 & 1576 & 1293 & 1211 & 1050 & - & - \\
\hline Ho1 & 3288 & 1587 & 1537 & 1287 & 1219 & 1052 & - & 463 \\
\hline HL2 & 3275,3100 & 1620 & 1587 & 1296 & 1205 & 1052 & - & - \\
\hline Ho2 & 3364 & 1585 & 1526 & 1294 & 1225 & 1057 & - & 464 \\
\hline HL3 & $3412,3375,3100$ & 1604 & 1577 & 1286 & 1211 & 1050 & 694 & - \\
\hline Ho3 & 3177 & 1581 & 1520 & 1290 & 1224 & 1059 & 691 & 465 \\
\hline HL4 & $3325,3200,3100$ & 1623 & 1589 & 1287 & 1210 & 1055 & 693 & - \\
\hline Ho4 & 3354 & 1580 & 1519 & 1286 & 1223 & 1060 & 689 & 466 \\
\hline HL5 & $3325,3187,3100$ & 1620 & 1585 & 1286 & 1224 & 1051 & 692 & - \\
\hline Ho5 & 3386 & 1582 & 1519 & 1285 & 1222 & 1053 & 680 & 472 \\
\hline
\end{tabular}

$\mathrm{X}=$ halogen

The (C-O) stretching frequency for the ligand is found at $1210 \mathrm{~cm}^{-1}$, which is shifted to the higher frequencies by $10-35 \mathrm{~cm}^{-1}$ in the complexes, which indicates coordination through the phenolato oxygen. The (N-O) stretching frequency is detected at $1050 \mathrm{~cm}^{-1}$ which showed a notable shift to a higher frequncy region by $5-20 \mathrm{~cm}^{-1}$ after complexation, confirming the coordination through the oximino nitrogen (Piperpont et al)[11]. Absorption at $688-695 \mathrm{~cm}^{-1}$ is assigned to (C-X); after complexation this band is shifted to lower region by $3-10 \mathrm{~cm}^{-1}$.

A band at $460-480 \mathrm{~cm}^{-1}$ of medium intensity is observed in the spectra of complexes which is assigned to the (Ho-O) stretching band. All the complexes showed slightly smaller magnetic moments than the calculated value of free Ho (III) ion (10.6 BM) suggesting that $4 \mathrm{f}$ electrons in these compounds do not participate in complex formation.

\section{Antimicrobial activity of the ligands and their metal chelates}

No zone of inhibition of growth was observed in any of the culture plates against any of the compound by the end of $48 \mathrm{~h}$, showing that organisms tolerate and survive the disk concentrations $(30 \mu \mathrm{g})$. So the concentrations of compounds used for MIC were more than 30 
$\mu \mathrm{g} / \mathrm{mL}$. The MIC values of all the compounds tested against various microorganisms is shown in table 4. It is apparent from table 4 that the MIC values are very high for each organism, explaining the extent of resistance offered by them, against these compounds.

Table 4. Antimicrobial activity (MIC in $\mu \mathrm{g} / \mathrm{mL}$ ) of ligands and their metal complexes against various microorganisms.

\begin{tabular}{|l|l|l|l|l|l|l|l|l|l|}
\hline Compd & 1 & 2 & 3 & 4 & 5 & 6 & 7 & 8 & 9 \\
\hline HL1 & $<512$ & $>1024$ & 512 & $>1024$ & $>1024$ & $>1024$ & $>1024$ & $<512$ & $>1024$ \\
\hline HL2 & 256 & $>1024$ & 512 & $>1024$ & 256 & $>1024$ & $>1024$ & $>1024$ & $>1024$ \\
\hline HL3 & $>1024$ & $>1024$ & $>1024$ & $>1024$ & 512 & $>1024$ & $>1024$ & 512 & $>1024$ \\
\hline HL4 & 256 & 256 & $>1024$ & $>1024$ & 512 & $>1024$ & $>1024$ & 512 & $>1024$ \\
\hline HL5 & $>1024$ & $>1024$ & $>1024$ & $>1024$ & 256 & $>1024$ & $>1024$ & $<512$ & $>1024$ \\
\hline Ho1 & 256 & 512 & 256 & $>1024$ & 512 & $>1024$ & $<512$ & 128 & $>1024$ \\
\hline Ho2 & 128 & $>1024$ & 256 & $>1024$ & 128 & $>1024$ & $>1024$ & $>1024$ & $>1024$ \\
\hline Ho3 & $>1024$ & $>1024$ & $>1024$ & $>1024$ & $<128$ & $>1024$ & $>1024$ & 128 & $>1024$ \\
\hline Ho4 & 128 & $<128$ & 512 & $>1024$ & $<128$ & $>1024$ & $>1024$ & 256 & 128 \\
\hline Ho5 & $>1024$ & $>1024$ & $>1024$ & $>1024$ & 512 & $>1024$ & $>1024$ & $<256$ & $>1024$ \\
\hline
\end{tabular}

HL, ligand; Ho, metal complex; 1, Acinetobacter; 2, Proteus; 3, Salmonella; 4, Klebsiella; 5, S. aureus; 6, Pseudomonas; 7, E.coli; 8, E. coli HB101; 9, Providencia.

$S$. aureus is fairly sensitive to all compounds except lawsonemonoxime, when compared with other organisms tested. Acinetobacter was showing moderate MIC values against lawsonemooxime, phthiocolmonoxime, Ho(III) lawsonemonoximate, Ho(III) bromolawsonemonoximate; while against all other compounds, the MIC was more than 1024 $\mu \mathrm{g} / \mathrm{mL}$. This may explain an inherited resistance of these organisms for these compounds. The chelates namely, Ho(III) lawsonemonoximate, Ho(III) chlorolawsonemonoximate, Ho(III) bromolawsonemonoximate, and Ho(III) iodolawsonemonoximate shown excellent antimicrobial activity against $E$. coli; wherein the organism was resistant to their corresponding ligands (table 4). Acinetobacter had also a moderate antimicrobial activity against metal complexes of the ligands, to whom the organism was earlier resistant. So we presumed that the antimicrobial activity is due to bonding of metal complexes between the atom of metal ion and the enzymes of microbial cell; which must be leading to inhibition of microbial growth. The exact mechanism of activity needs to be studied carefully. But looking at further uses of $f$ block (rare earth) series in biological systems, this study may turn helpful in the near future.

\section{ACKNOWLEDGEMENT}

This work was supported by a Department of Chemistry, University of Pune, through a University Grants Commission's minor research project. The author thank Department of Chemistry, University of Pune; \& Department of Microbiology and Pathology of DSH Medical College \& Hospital for the facilities provided to carry out this work.

\section{REFERENCES}

1. Lime, O.G. de, Coelho, J. S., Albuquerque, I.L de, Mello, J. F. de, Martin, D. G., Lacerda, A. L., Moraes Souza, M. A. de., Rev. Inst. Antibiot. (Recife), 11, 21-26 (1971).

2. Tripathi, R. D., Srivastava, H. S., Dixit, S. N., Experientia, 34, $51-52$ (1978).

3. Anderson, R. J., Newman, M. S., J. Biol. Chem., 101, 773 (1933).

4. Mhaske, R. B., Kulkarni, M., Joshi, S. B., Kshirsagar, V. G., Pradhan, S. P. Ind. J. Pharm. Sci., 61(5), 293-296 (1999).

5. Dandawate, F. V., Kodolikar, J. G., Kelkar, V. D., Kulkarni, B. A., Themochim. Acta, 241, 103 (1994).

6. Gaikwad, S. D., Ph. D. Thesis, University of Pune (India), (1997).

7. Joshi, C., R., Jagtap, G. S., Chalgeri, S. V.. Ind. J. Pharm. Sci., 49, 188-190 (1987).

8. Shen Xu, Li , Jiang, H., Xie, Y. Synth. React. Inorg. Met. Org. Chem. 25, $1239-47$ (1995)

9. Fieser, L. F., J. Biol. Chem., 133, 391 (1940).

10. National Committee for Clinical Laboratory Standards, NCCLS documents, M2-A4 (1990).

11. Piperpont, C. G., Downs H. M., Rukavina, T. G., J. Am. Chem. Soc. 96, 5573 (1974).

Received: May 30, 2000 - Accepted: June 6, 2000 Received in publishable format: June 9, 2000 